Molecular Physics, 2014

Vol. 112, No. 1, 127-141, http://dx.doi.org/10.1080/00268976.2013.804960

\title{
Thermodynamic properties and the approximate solutions of the Schrodinger equation with the shifted Deng-Fan potential model
}

\author{
K.J. Oyewumia,*, B.J. Falayea, C.A. Onatea, O.J. Oluwadareb and W.A. Yahyac \\ ${ }^{a}$ Theoretical Physics Section, Department of Physics, University of Ilorin, P. M. B. 1515, Ilorin, Nigeria; \\ ${ }^{b}$ Department of Physics, Federal University Oye-Ekiti, P. M. B. 373, Ekiti State, Nigeria; \\ ${ }^{c}$ Department of Physics and Material Science, Kwara State University, P. M. B. 1530, Ilorin, Nigeria
}

(Received 28 February 2013; final version received 8 May 2013)

With the introduction of a new improved approximation scheme (Pekeris-type approximation) to deal with the centrifugal term, the energy eigenvalues and the wave functions of the Schrodinger equation of the shifted Deng-Fan molecular potential " are obtained, via the asymptotic iteration method. Rotationalvibrational energy eigenvalues of some diatomic molecules are presented, these results are in good agreement with other results in the literature. For these selected diatomic molecules, energy eigenvalues obtained are in much better agreement with the results obtained from the rotating Morse potential model for moderate values of rotational and vibrational quantum numbers. Furthermore, thermodynamic properties such as the vibrational mean $U$, specific heat $C$, free energy $F$ and entropy $S$ for the pure vibrational state in the classical limit for these energy eigenvalues are studied.

Keywords: shifted Deng-Fan molecular potential; Pekeris-type approximation; asymptotic iteration; eigenvalues;

Molecular Physics: An International Journal at the Interface Between Chemistry and Physics, 112:1, 127141, DOI: 10.1080/00268976.2013.804960 